\title{
La résilience au fil de l'eau
}

Le concept de résilience est, pour les uns, une métaphore aussi séduisante qu'inutile, un simple mot à la mode, voire une manière déguisée de nous apprendre à encaisser des coups sans broncher. Pour les autres, " penser résilience », c'est au contraire s'inscrire dans le changement, aller de l'avant, s'adapter à un monde incertain, et admettre que les leçons du passé ne répondent pas toujours aux enjeux du futur. Il y a là matière à philosopher, mais tel n'est pas, loin s'en faut, le seul objet de ce concept.

\section{La réalité forestière n'est plus un long fleuve tranquille.}

Le changement climatique produit déjà de nouveaux régimes de perturbation, plus intenses et imprévisibles, modifiant les assemblages d'espèces, facilitant les pullulations de ravageurs. Les politiques commerciales et environnementales se modifient à grand train en réponse à des attentes capricieuses. Le monde se façonne et s'emballe au rythme d'événements extrêmes et soudains, sans grands égards aux régimes moyens. Les digues s’ébranlent.

Dans un environnement global versatile et un contexte économique aussi brutal, maintenir les forêts dans un état optimal devient paradoxalement hasardeux. Maximiser la production d'une gamme restreinte de biens ou de services, agir « du mieux possible » sur les facteurs considérés comme les plus appropriés, voilà qui s'annonce aujourd'hui illusoire et périlleux. Plus déroutant encore, les décennies de capitalisation d'expériences sylvicoles apparaissent comme hésitantes et impuissantes face aux réalités inédites des écosystèmes forestiers d'aujourd'hui et de demain.

La résilience n'est certes pas une panacée permettant de lever autant de difficultés et de maintenir le cap sans avaries, mais elle peut être utile pour éviter qu'une brèche ne se fasse jour sous la coque du navire. De quoi s'agit-il au juste ? La résilience est définie comme la capacité d'un système à absorber une perturbation sans modifier radicalement ses caractères fonctionnels et structuraux. Il y faut de la souplesse. Or, optimiser un système, c'est précisément le rendre peu déformable, peu flexible, et de ce fait fragile et davantage enclin à se percer, à prendre l'eau.

Penser résilience, c'est à l'inverse gérer les systèmes en les inscrivant dans le fil du changement, sans résister ni cependant tout à fait céder. Cela impose de garder constamment un œil sur les seuils de rupture au-delà desquels le système peut basculer, et les éviter. Sinon, le

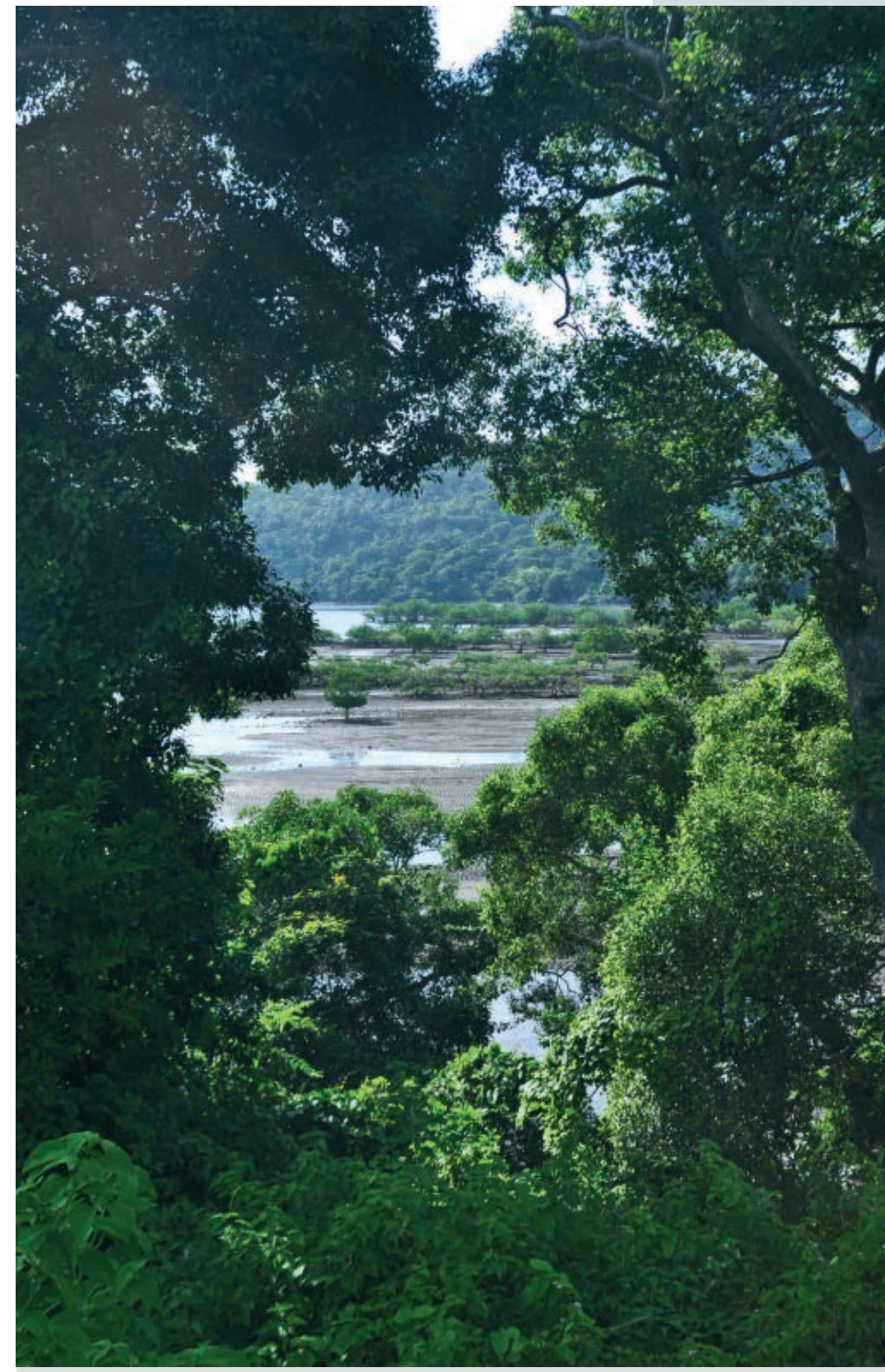

Gérer les écosystèmes en les inscrivant dans le fil du changement.

Photo J. Tassin. 
choc peut s'avérer brutal et donner lieu à des transformations profondes, voire spectaculaires. Ainsi les savanes, les forêts humides ou les espaces dénudés d'arbres virent-ils radicalement d'une forme à l'autre en fonction de seuils de précipitations précis, au demeurant universels ${ }^{1}$. Cela advient rarement sans dommages.

En premier lieu, considérer la résilience d'une forêt impose de considérer le fonctionnement de celle-ci dans la dimension d'un socio-écosystème. L'adaptation au changement met en effet en œuvre des processus autant écologiques qu'humains. C'est aussi devoir repérer les processus clés qui dynamisent ou altèrent cette résilience. Par exemple, il s'agit de connaître les groupes fonctionnels de réponse aux principales perturbations, et leurs comportements spécifiques face aux changements. Penser résilience, c'est aussi en finir avec les mythes ou métaphores invitant à regarder la nature sous l'angle réducteur du prisme organismique. Contrairement aux idées reçues, un écosystème forestier n'est pas un organisme vivant. Il n'est ni stable, ni homéostatique, ni voué à un destin climacique quelconque, et ne se reproduit pas à l'identique.

\section{Comment dès lors gérer la résilience forestière ?}

Il s'agit d'éviter les seuils de rupture évoqués précédemment, de louvoyer entre eux ou de les rendre plus difficilement atteignables.

Le meilleur gouvernail demeure l'innovation. Il s'agit par exemple, en adaptant les règles sylvicoles aux perspectives de changement, de maintenir une diversité de réponses possibles à l'incertain, et de préserver ainsi une garantie sur l'avenir. Utiliser des essences relevant de modes de régénération divers, diversifier les prescriptions sylvicoles, prévoir des mélanges d'essences associant plusieurs groupes fonctionnels, privilégier les espèces tolérantes aux épisodes de sécheresse en minimisant de ce fait les espèces pionnières ${ }^{2}$, varier les écartements tout en intensifiant les éclaircies afin de redistribuer la ressource en eau auprès d'un nombre moins élevé de tiges, accroître l'hétérogénéité des plantations, diversifier les rotations de coupe des monocultures : telles sont quelques-unes des nombreuses pratiques forestières au service d'une indispensable flexibilité.

\footnotetext{
1 De tels seuils de basculement ont été tout récemment mis en évidence par une équipe de recherche de l'Université de Wageningen.

2 Voir la thèse de D. OUÉADRAOGo (2011), qui montre qu'en République centrafricaine, sur le dispositif de M'Baiki, les espèces pionnières se montrent les plus sensibles aux périodes de sécheresse.
}

Mais la résilience forestière procède aussi de l'aménagement quand il s'agit de préserver la connexion spatiale et fonctionnelle au sein d'un massif naturel ou planté. Là encore, on peut énumérer bien des stratégies possibles pouvant s'avérer plus ou moins souhaitables selon les contextes rencontrés : favoriser si nécessaire le développement d'un sous-bois, conserver des zones tampons plus ou moins régulièrement réparties, promouvoir des bandes de végétation naturelle afin d'assurer une meilleure régulation biotique, protéger les vertébrés disperseurs frugivores, promouvoir les flux d'espèces avec d'autres unités de paysage, etc.

Encore un mot, pour finir, sur les systèmes agroforestiers, parfois portés au pinacle des modèles durables ; ceux-ci n'échappent pas davantage à la crue des changements globaux. Or, les systèmes les plus intra-dépendants et les moins modulaires sont souvent peu résilients. Leurs combinaisons spatiales, optimales au regard des conditions actuelles, ne le seront peut-être plus demain dans une perspective de raréfaction des précipitations. Leurs composantes interactives garantissent certes plus d'efficacité, plus d'autonomie, mais elles souffrent de ce fait d'un déficit de souplesse. Trop fermement ajustés, les systèmes agroforestiers peuvent eux aussi voir leurs coutures mises à mal en présence de changements brutaux. Alors, gare aux voies d'eau : aucun colmatage ne tiendra.

Innovation, adaptation, diversité, flexibilité, modularité : ainsi s'énumèrent quelques-unes des clés de la résilience. Penser résilience forestière, c'est, équipé d'un tel trousseau, s'embarquer, descendre au fil des rapides contemporains, débarquer parfois pour éviter les seuils trop périlleux, se prémunir des écueils. Et surtout, regarder loin devant.

\section{Jacques Tassin}

Membre du Comité de rédaction de Bois et forêts des tropiques.

Chercheur en écologie végétale au Cirad, dans l'unité de recherche Biens et services des écosystèmes forestiers tropicaux.
HIROTA M., HOLMGREN M., VAN NES E. H., SCHEFFER M., 2011. Global resilience of tropical forest and savanna to critical transitions. Science, 334: 232-235.

OUÉDRAOGO D., 2011. Prédiction de la dynamique forestière à l'aide d'un modèle matriciel qui incorpore la variabilité de la réponse des espèces à l'environnement : application dans une forêt tropicale humide semi-décidue d’Afrique centrale. Thèse, Université Montpellier 2. 